\title{
Theoretical investigation of the effect of sample properties on the electron velocity in quantum Hall bars
}

\author{
D. Eksi, E. Cicek, A. I. Mese, and S. Aktas \\ Department of Physics, Faculty of Arts and Sciences, Trakya University, 22030 Edirne, Turkey
}

A. Siddiki

Arnold Sommerfeld Center for Theoretical Physics, Physics Department and Center for NanoScience, Ludwig-Maximilians-Universität, Theresienstrasse 37, 80333 Munich, Germany

T. Hakioğlu

Department of Physics and National Center for Nanotechnology Research, Bilkent University, 06690 Ankara, Turkey (Received 20 December 2006; revised manuscript received 27 February 2007; published 21 August 2007)

\begin{abstract}
We report on our theoretical investigation of the effects of the confining potential profile and sample size on the electron velocity distribution in (narrow) quantum Hall systems. The electrostatic properties of the electron system are obtained by the Thomas-Fermi-Poisson nonlinear screening theory. The electron velocity distribution as a function of the lateral coordinate is obtained from the slope of the screened potential at the Fermi level and within the incompressible strips. We compare our findings with the recent experiments.
\end{abstract}

DOI: 10.1103/PhysRevB.76.075334

PACS number(s): 73.20.-r, 73.50.Jt, 71.70.Di

\section{INTRODUCTION}

In the early electro-optical measurements performed on the two-dimensional electron systems (2DESs), the electrostatic potential across the Hall bar was shown to exhibit local dips as a function of the lateral coordinate across the sample. ${ }^{1-3}$ The positions of these local potential variations are strongly dependent on the applied perpendicular magnetic field. They coincide with the positions of the strips with finite width corresponding to integer local filling factors where the longitudinal conductance vanishes, i.e., $\sigma_{\ell}(x)$ $=\sigma_{x x}(x)=\sigma_{y y}(x)=0$. It was concluded that the "expected" quasi-one-dimensional edge states can be as large as hundreds of micrometers, where $\sigma_{\ell}(x) \neq 0$. It was also shown that the current can flow from the bulk in the magnetic field interval where the Hall resistance does not assume its quantized value. In this regime, however, the outermost edge states are reported to be "invisible." 3 On the other hand, the local probe of the electrostatic potential and the longitudinal resistivity measurements ${ }^{4-7}$ using scanning force microscopy and single-electron transistor has indicated that the current is confined within finite regions across the sample. These regions were later suggested to be the "incompressible" regions, namely, regions of integer local Landau filling factors which are distributed in an inhomogeneous manner over the sample due to the electronic nonlinear screening as well as the boundary effects as previously predicted. ${ }^{8,9}$ These experiments are well explained by the recent theoretical works, ${ }^{10-12}$ which take into account interaction effects by exploiting the smooth confining potential profile within the Thomas-Fermi approximation and also incorporating a local version of the Ohm's conductivity model. These models contribute not only to the understanding of the induced electric field and current distribution but also to the high precision nature of the lowtemperature integer quantized Hall $(\mathrm{QH})$ plateaus in narrow
Hall bars as a function of the continuous lateral sample coordinate.

Recently, the edge profile of a InP/InGaAs Hall sample was probed in the "surface photovoltage (SPV) spectroscopy" measurements, and it was found that the electron velocity at the edges increases with increasing magnetic field $(B)$ respecting a square root behavior, ${ }^{13}$ i.e., $v_{\mathrm{el}}(B) \propto B^{1 / 2}$. In the interpretation of the data, these authors used a model in which the electrostatic bending of the Landau levels (resulting from the confinement potential) was not taken into account, the electron Hartree potential was neglected, and instead, the velocity distribution was modeled by an homogeneous induced electric field. It was stated therein that these measurements should be reinterpreted in the light of a "screening model." It is the aim of the present paper to show that their original interpretation cannot be corrected even by including a linear screening model (see Sec. III). In parallel to these developments, the nonlinear screening was also promoted by the importance of the recent electronic-MachZehnder interferometer experiments, ${ }^{14,15}$ where the role of the electron-electron interaction on the $B$ field dependence of the edge fields was emphasized. In these latter experiments the electron phase deduced from roughly assuming a constant group velocity of $v_{g} \sim(2-5) \times 10^{6} \mathrm{~cm} / \mathrm{s}$ disagrees with the single particle picture, and the authors argued this in favor of a possible breakdown of the single particle picture and the Landauer-Büttiker conductance formalism.

In the present work, we systematically analyze the electrostatic edge profile of narrow Hall bar samples using a self-consistent Thomas-Fermi-Poisson approach under $\mathrm{QH}$ conditions. In Sec. II, we introduce a model which incorporates a constant donor density in the manner of the Chklovskii geometry ${ }^{16}$ and, secondly, (Sec. III) by a nonuniform distribution of donors. We then find, in Sec. III and Sec. IV, the electron velocity $v_{y}$, considering different models, across the sample in the current direction by 


$$
v_{y}=\frac{1}{\hbar} \frac{\partial E_{X, n}}{\partial k_{y}},
$$

where $E_{X, n}$ is the eigenenergy of the single particle Hamiltonian with $X=\hbar k_{y} / e B$ denoting the center coordinate and $n$ the Landau Level (LL) index. Here, $k_{y}$ is the conserved electron momentum in the $y$ direction, $e$ is the electron charge, and $B$ represents the strength of the perpendicular magnetic field. We then investigate the dependence of the electron velocities on the $B$ field considering two edge state models in Sec. IV. The widths of the incompressible strips (ISs) depending on the sample properties are examined in Sec. V. We observe that the electron transport is confined within the ISs where the electron velocity decreases with increasing magnetic field as $B^{-1 / 2}$. On the other hand, if the center filling factor $\nu(0)$ is smaller than its minimum integer value of 2 (since we do not resolve the spin degeneracy), all the current is spread over the sample, suggesting that the slope of the screened potential should be calculated at the Fermi level. We close our discussion with a summary section.

\section{MODEL}

The 2DES, described by the electron number density $n_{\mathrm{el}}(x)$, is considered to be in the $x-y$ plane with a lateral confinement $|x|<b$ at $z=0$ and assuming translation invariance in the $y$ direction. The ionized donors also reside in this plane, with the average number density $n_{0}$ confined into the interval $|x|<d$, where $d$ is the sample width and $(d-b)$ the depletion length with $b<d$. Electrostatic self-consistent solution is then independent of the $y$ coordinate, and from the solution of the Poisson's equation with the appropriate boundary conditions, i.e., $V(x= \pm d, z=0)=0$, we obtain the Hartree potential energy of an electron in the plane of the 2DES as

$$
V_{H}(x)=\frac{2 e^{2}}{\bar{\kappa}} \int_{-d}^{d} d x^{\prime} K\left(x, x^{\prime}\right) n_{\mathrm{el}}\left(x^{\prime}\right),
$$

with $\bar{\kappa}$ being the dielectric constant of the material and the electrostatic kernel $^{9}$ is

$$
K\left(x, x^{\prime}\right)=\ln \left|\frac{\sqrt{\left(d^{2}-x^{2}\right)\left(d^{2}-x^{\prime 2}\right)}+d^{2}-x^{\prime} x}{\left(x-x^{\prime}\right) d}\right| .
$$

The potential energy of an electron in the confinement region generated by the donors reads

$$
V_{\mathrm{bg}}(x)=-E_{0} \sqrt{1-(x / d)^{2}}, \quad E_{0}=2 \pi e^{2} n_{0} d / \bar{\kappa},
$$

which can be found from Eq. (2) using the kernel given in Eq. (3) and replacing $n_{\mathrm{el}}\left(x^{\prime}\right)$ by $-n_{0}$. We write the total potential energy of an electron as $V(x)=V_{H}(x)+V_{\mathrm{bg}}(x)$. The electron number density is calculated numerically, within the Thomas-Fermi Approximation (TFA),

$$
n_{\mathrm{el}}(x)=\int d E D(E) f\left([E+V(x)-\mu] / k_{B} T\right),
$$

with $D(E)$ describing the (collision-broadened) Landau density of states (DOS), $f(\alpha)=\left(1+e^{\alpha}\right)^{-1}$, the Fermi distribution function, and $\mu$ the electrochemical potential. Here, $k_{B}$ and $T$ represent the Boltzmann constant and the electron temperature, respectively. We also assume the electron spin degeneracy. Equations (2) and (5) complete the self-consistent scheme, ${ }^{10,11}$ which can be solved by a numerical iteration. For accurate convergence, we first perform calculations at $T=0$ and $B=0$, then increase $T$ at an elevated $B$ strength, and reduce the temperature stepwise until the relevant temperature is achieved. In the next sections, we first consider two distribution functions for the donor number density and investigate the electron velocity dependence on the magnetic field, temperature, and confining potential profile.

\section{DONOR DISTRIBUTION}

During the last decades, several boundary conditions were considered, ranging from infinite hard-wall potentials ${ }^{17}$ to smooth potentials ${ }^{9,16,18}$ in order to theoretically investigate the 2DES under $\mathrm{QH}$ conditions. For relatively large samples $(d \gtrsim 1 \mathrm{~mm})$, the edge effects were considered to be dominated by the localization; thus, the positions of the ISs were mainly predicted by the disorder potential. On the other hand, for narrow samples $(d<15 \mu \mathrm{m})$, the ISs were considered to be formed due to the electrostatic boundary conditions at the edges. Recently, it was experimentally shown that the steep potential at the edge of the sample prohibits the formation of the ISs and the Chklovskii picture is no longer applicable. ${ }^{19}$ These results coincide with an early theoretical calculation, based on Hartree approximation, given in Ref. 17, where the edge potential is taken to be an infinite wall, for which ISs were not observed. On the other hand, if the edge profile is smooth, several incompressible regions can be observed theoretically, ${ }^{9,18,20}$ which are confirmed experimentally. ${ }^{21}$ In the intermediate case, corresponding to narrow samples, only a single incompressible edge strip was reported, ${ }^{4,5}$ which was then supported by subsequent theoretical works. ${ }^{11,22}$

In this section, we consider narrow samples $(1 \mu \mathrm{m} \lesssim d$ $\lesssim 5 \mu \mathrm{m})$ and vary the donor distribution to investigate the widths of the ISs depending on the magnetic field strength. The selected donor profiles can be realized experimentally either by the uncontrollable etching processes or by gradually doping the sample.

In Fig. 1, we show the two selected donor distributions (upper panel) and the corresponding confinement potentials (lower panel) generated by

$$
\rho_{1}(u)= \begin{cases}\frac{\left[-(u+c)^{2}+(c-1)^{2}\right] n_{c}}{(c-1)^{2}}, & -1 \leqslant u<-c \\ \frac{n_{c},}{\frac{\left[-(u-c)^{2}+(1-c)^{2}\right] n_{c}}{(c-1)^{2}},} & -c<u \leqslant c\end{cases}
$$




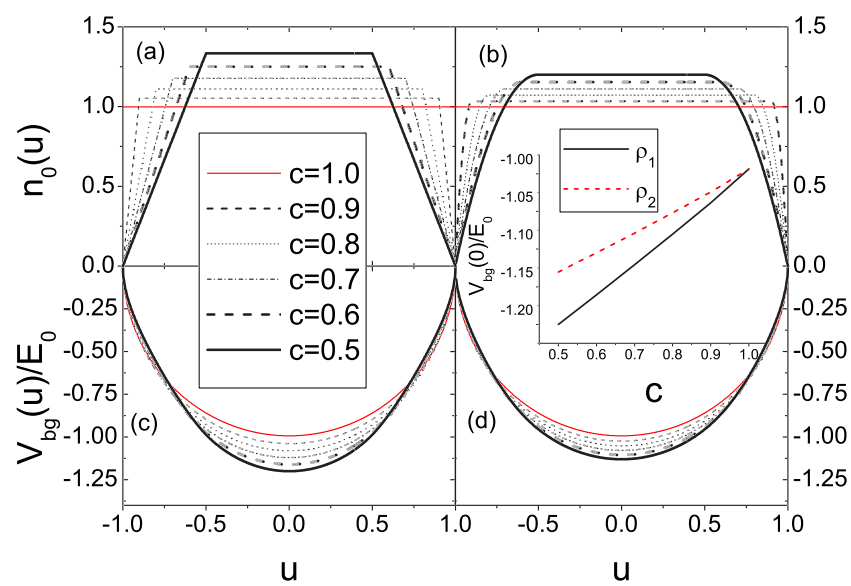

FIG. 1. (Color online) The cross section of the donor layer considering (a) $\rho_{1}(u)$ and (b) $\rho_{2}(u)$ for various values of the steepness parameter $(0.5 \leqslant c \leqslant 1)$, together with the calculated background potential profiles [lower panels, (c) and (d)]. The thin solid line represents a constant donor distribution $(c=1)$, whereas thick solid line corresponds to $c=0.5$. The line code denotes a gradual increase of $c$ with a step of $10 \%$. The sample width $d$ and the depletion length $b$ are fixed and set to be $3 \mu \mathrm{m}$ and $300 \mathrm{~nm}$, respectively. In both cases, the donor number density is kept constant and chosen to be $4 \times 10^{11} \mathrm{~cm}^{-2}$. The inset depicts the variation of the background potential calculated at the center of the sample for $\rho_{1}$ (thin solid line) and $\rho_{2}$ (broken line).

$$
\rho_{2}(u)= \begin{cases}\frac{(u+1) n_{c}}{(1-c)}, & -1 \leqslant u<-c \\ n_{c}, & -c \leqslant u \leqslant c \\ \frac{(1-u) n_{c}}{(1-c)}, & c<u \leqslant 1,\end{cases}
$$

where $u=x / d$ and $n_{c}$ is a constant density preserving the total number of the donors in the sample. The steepness of the confinement is controlled by the dimensionless parameter $c$. In the figures, the potential energies are also normalized with the pinch-off energy $\left(E_{0}\right)$ of the constant donor distribution. To make a connection between the experimental realizations of such donor distributions, we point out that, during the chemical etching in the $z$ direction, the reaction also takes place in the $x-y$ plane. Hence, the donor layer is not necessarily etched completely at the edges and a distribution similar to $\rho_{1}(u)$ is expected. Meanwhile, during the growth process of the wafer, donors can be distributed (in a controlled way) similar to $\rho_{2}(u)$. In our calculations in both cases, we keep the average donor number density constant. It is clearly seen in Fig. 1 that the steepness profiles close to the edges of the sample are different for two distribution functions, whereas the minima of the confining potentials change linearly with $c$. As a result, different behaviors can be identified for the screened potentials (even without exploiting the magnetic field) arising from the momentum $(q)$ dependence of the Thomas-Fermi dielectric function, i.e., $\epsilon(q)=1+\frac{2}{a_{B}^{*}|q|}$ dominated by $q=2 \pi / a$.

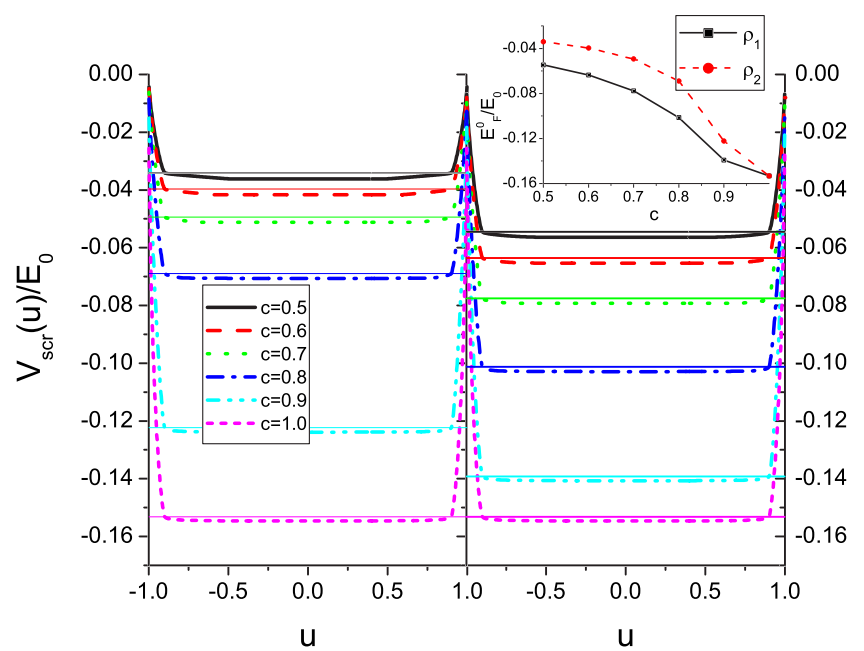

FIG. 2. (Color online) The screened potentials obtained from the bare confinement potentials shown in Fig. 1, at $B=0$ and $T=0$ (thick lines) and also the Fermi energy for vanishing field and temperature (thin horizontal lines). The inset depicts the variation of $E_{F}^{0}$ versus the steepness considering $\rho_{1}$ (solid line) and $\rho_{2}$ (broken line).

The relation between the screened potential $V_{\text {scr }}(q)$ and the external potential $V_{\text {ext }}(q)$ is given by

$$
V_{\mathrm{scr}}(q)=V_{\mathrm{ext}}(q) / \epsilon(q) \text {. }
$$

Different steepness values lead to different dominating $q$ regions, which then render different characteristic screening properties. From the inset of Fig. 1, we conclude that the steepness of the potential increases much faster for $\rho_{1}$, better simulating the edge profile than the doped profile. This difference becomes crucial when a magnetic field is applied and the ISs are formed at the edges of the sample.

In the next step, we consider the effect of the electronic screening. We calculate the screened potential selfconsistently by solving Eqs. (2) and (5) at $T=0$ and $B=0$. In this limit, Eq. (5) is reduced to

$$
n_{\mathrm{el}}(x)=D_{0}\left(E_{F}^{0}-V(x)\right) \Theta\left(E_{F}^{0}-V(x)\right),
$$

which then becomes a linear relation between the potential and the electron distribution within the linear screening regime. In Fig. 2, the calculated self-consistent potentials are depicted for the considered donor distributions together with the variation of the Fermi energy as a function of $c$. On one hand, the screened potential within the sample coincides with the $E_{F}^{0}$ for both models in Eq. (6) and (7). On the other hand, these quantities differ strongly for both distributions due to the nonlinear screening pronounced above. Since the constant part of the donor distribution $(q=0$ component) is strongly screened, the change of the potential depending on steepness is less pronounced for $\rho_{2}(x)$; meanwhile, the sharp transition is observed at $\rho_{1}(x)$. This implies that more $q$ components are involved in the screening there. This behavior can be seen from the slope shown in the inset of Fig. 1. In other words, less $q$ components contribute, for $\rho_{1}(x)$, to the screened potential in the bulk, whereas more $q$ components are involved close to the edge of the sample. Thus, the mini- 


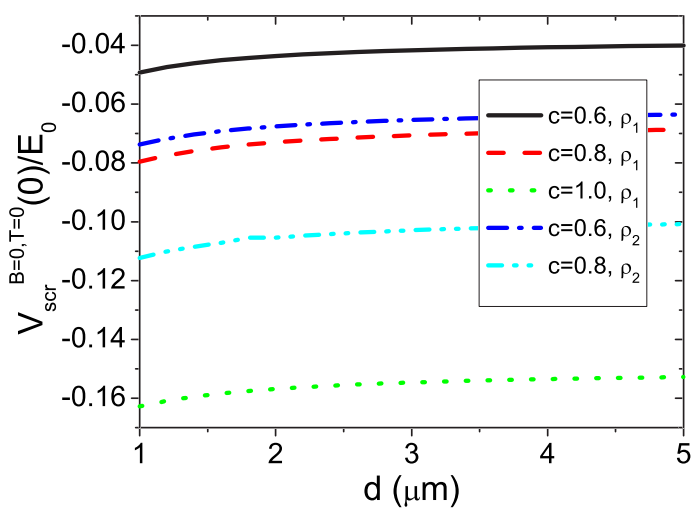

FIG. 3. (Color online) The sample width dependence of the screened potential calculated at the center. The line code depicts the selected values of $c$ and two distribution functions.

mum of the screened potential changes faster than that of $\rho_{2}(x)$. The Fermi energies show a similar behavior, up to a factor, which indicates that the average number of electrons decreases faster for $\rho_{1}(x)$ although the density of the donors is kept constant.

The effect of the sample width on the potential profile affects the variation of the screened potential. In Fig. 3, the self-consistent potential at the center is plotted against the (half) sample width for the two donor distributions in Eqs. (6) and (7) for the selected steepness parameters. For large samples $(2 d \geqslant 6 \mu \mathrm{m})$, the variation of the central value of the potential is not sensitive to the steepness, since the electrons at the bulk can perfectly screen the confinement potential at the edges. On the other hand, steepness is expectedly important for narrower samples. This observation clarifies the dominating role of the edge profile on the electron velocity for narrow samples and shows that the interaction effects become important in the Mach-Zehnder-type experiments, where the dimensions of the samples used are usually less than a few micrometers $(2 d<3-4 \mu \mathrm{m})$.

Before proceeding with the investigation of the effects of the magnetic field, i.e., considering the effects of the IS in the presence of nonlinear electronic screening, we briefly discuss the slope of the self-consistent Hartree potential by only taking into account the LL quantization. As the magnetic field is changed, the LLs and their separations are shifted on the energy axis. A qualitative understanding of the $B$ dependence of the induced electric field $(F)$ within the sample can then be achieved by analyzing this shift. Then, $F(B)$ can be compared with the experimental results obtained by Ref. 13 .

Our main argument is that, even without taking the ISs into account, one should be able to observe the predicted behavior of the (average) electron velocity $\left(v_{\mathrm{el}}\right)$ at the edges of the sample. Since in their model the electron-electron interaction (also without magnetic field) was not taken into account, therefore their results should be a limiting case of our first order approximation. This argument relies also on the fact that, within the single particle picture, the electron velocity is calculated from the slope of the LLs (essentially from the confinement potential) at the Fermi level. Since in

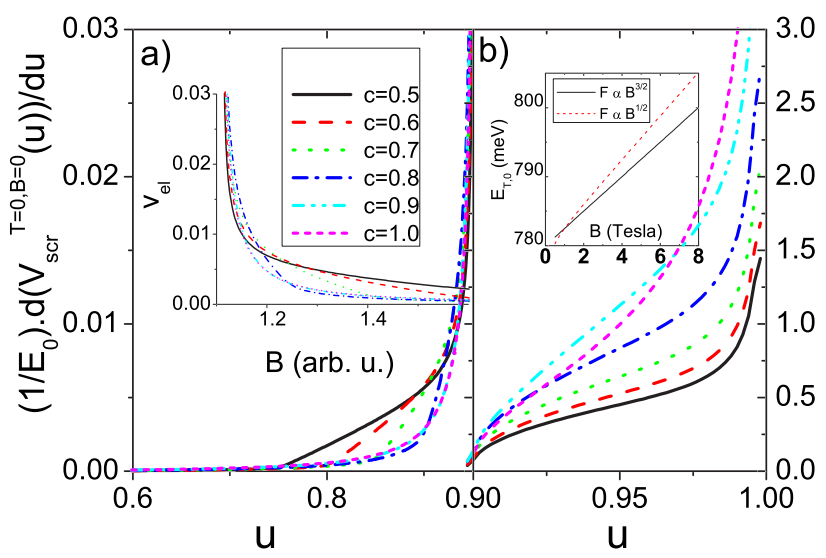

FIG. 4. (Color online) The numerical derivative of the screened potentials for different steepness values calculated at the edge of the sample for inverse parabolic donor distribution $\rho_{2}$. Horizontal axis essentially presents the Fermi level, i.e., increasing of the $u$ corresponds to increasing of the average electron density.

the Landauer-Büttiker ${ }^{23}$ formalism the electron velocity plays no role due to cancellation of the velocity with the arguments of the one-dimensional DOS, the simplest next step would be to take into account the screening without magnetic field and rewrite the energy dispersion within the TFA, yielding

$$
E_{X, n}=E_{n}+V_{\mathrm{scr}}^{T=0, B=0}(X),
$$

where $E_{n}$ is the Landau energy $\left[=\hbar \omega_{c}(n+1 / 2)\right]$ and $V_{\text {scr }}^{T=0, B=0}(X)$ the screened potential calculated at vanishing magnetic field and temperature.

We use the derivative of this potential with respect to $u$ at the Fermi level to infer $v_{\mathrm{el}}$. This derivative of $V_{\mathrm{scr}}(u)$ is shown in Fig. 4 for selected values of $c$ and using $\rho_{2}(u)$ as the donor distribution. We observe two characteristic behaviors. In Fig. 4(a), in the electron dense region $(|u|<0.9$, where $\left.V_{\mathrm{scr}}(u) / E_{0}<E_{F} / E_{0}\right)$, the derivative grows in the positive $x$ direction rather slowly compared to that in the electron depleted region, as shown in Fig. 4(b). Screening is strong in the electron dense interior where the total potential is flat. Approaching the edge of the populated region, the number of electrons decreases and screening becomes poor. In the depleted region, the confinement potential is screened very poorly; thus, the variation of the total potential there is large yielding a larger derivative. For a given Fermi energy, decreasing the magnetic field corresponds to sweeping the $x$ axis by which the electron velocity along $y$ can be deduced. In the inset of Fig. $4, v_{\mathrm{el}} \propto B^{-1 / 2}$ behavior is clearly observed for all $c$ values; however, the exact quantitative values depend on the steepness of the edge profile. We observe that, for $c \leqslant 0.8$, the change in the electron velocity is much more rapid in this case than the shallow edge profiles, indicating a strong relation between the edge profile and the magnetic field dependence of the electron velocity. Here, we would like to focus on the crossover between the two different slope behaviors, namely, the $c \leqslant 0.8$ and $c>0.8$. If the potential slope is sharper at the edge, the local electron density at the 
edges reaches the bulk (average) density value much closer to the end of the sample; hence, the screened potential varies rapidly in the length scale of electron poor region, resulting in a high electron velocity. By electron poor region we mean the interval between the electron depleted stripe (see, e.g., Fig. 9 of Ref. $9,|x / d|>0.9)$ and where the local density is similar to the bulk value $(|x / d|<0.8)$. Considering the SPV experiments, one should expect the fast electron behavior, since the sample is gated from one side resulting in a steep profile, whereas in the Mach-Zehnder interference experiments, the boundary conditions are mixed, i.e., smooth near the quantum point contacts and steep near the chemically etched regions. For the shallow edge profile, the case is just the opposite, i.e., the electron poor region is much more extended (same figure of Ref. 9 for the solid lines 0.5 $<|x / d|<0.9)$ and therefore screening is not good as it was for the sharp edge, yielding a slower electron velocity. Combining $v_{\mathrm{el}} \propto B^{-1 / 2}$ and the calculated slopes demonstrates that, in the mentioned experiments, ${ }^{13}$ the confinement is relatively steep, which was concluded by these authors to be the opposite. In connection, here we would like to stress another experiment where a similar geometry reported in Ref. 19 was considered. In this work, it is ruled out that if a negatively charged gate is placed on the side perpendicular to the 2DES (in the experimental setup, this gate is another 2DES, obtained by a cleaved edge overgrowth technique), creating a sharp potential profile at this edge, no ISs are observed. Similarly, a side (gate) electrode is used to detect the SPV signal in the experimental setup of Karmakar et al. ${ }^{13}$ and their conclusion contradicts strongly with the findings of $\mathrm{Hu}-$ ber et al. ${ }^{19}$ They also contradict with the velocity dependence, which we discuss next in more detail now also including the incompressible regions. It is clear that we will work with those geometries where the edge profile is neither very steep (such as an infinite wall or a perpendicular side gate) nor very shallow so that many incompressible regions can be observed at a given magnetic field, within the Thomas-Fermi approximation.

In the SPV work, the Hamiltonian of the system was given by

$$
H=\frac{1}{2 m_{e}^{*}}(\mathbf{p}-e \mathbf{A})^{2}+e F x,
$$

which includes a constant electric field $(F)$ along the positive $x$ axis pointing to the edge. Here, $m_{e}^{*}$ is the effective electron mass and $\mathbf{p}$ and $\mathbf{A}$ are the canonical electron momentum and the vector potential, respectively. Using the Landau gauge, the energy dispersion is found to be $\mathrm{e}^{24}$

$$
E_{n, X}=E_{g}+(n+1 / 2) \hbar \omega_{c}-(F / B)\left(X / l_{b}^{2}\right)-\left(m_{e}^{*} / 2\right)(F / B)^{2},
$$

where $E_{g}$ is the energy band gap and $l_{b}=\sqrt{\hbar / m \omega_{c}}$ the magnetic length. These authors concluded that in order to obtain the $B=0$ value and also to match the experimental results (see Fig. 4 of Ref. 13), one should assume that $F \propto B^{3 / 2}$. First of all, one remark is that in the $B=0$ limit, energy dispersion given in Eq. (12) becomes meaningless. Secondly, in the limit of high magnetic field, assuming $F \propto B^{3 / 2}$ or $F \propto B^{1 / 2}$ essentially leads to similar linear behavior at the measured $B$ values, as shown in the inset of Fig. 4. In the SPV experiments, no low field $(B \leq 1 \mathrm{~T})$ measurement were performed; therefore, we conclude that their conclusion about $F \propto B^{3 / 2}$ in Ref. ${ }^{13}$ is not unique. Moreover, our calculations ascertain that, even in the absence of ISs, the electron velocity is an inverse square root function of the magnetic field, namely, $v_{\mathrm{el}} \propto B^{-1 / 2}$, and as a consequence, $F \propto B^{1 / 2}$.

Our simple self-consistent calculations, assuming that the effects of ISs are negligible, agree qualitatively well with the experimental findings. We also point that the functional form of the electric field and the interpretation of the steepness of the potential strongly differ from Ref. 13. First of all, it is experimentally ${ }^{19}$ and theoretically ${ }^{9,17}$ shown that in the presence of a side gate, perpendicular to the 2DES (simulating a hard-wall potential or surface charges), the potential at the edge is steep. Secondly, the proclaimed $B$ dependence of the electric field at the edge is not unique and we claim that $F$ $\propto B^{1 / 2}$.

The discussion above should also be reconsidered in the presence of IS. In the next section, we do that by examining both the potential slope at the Fermi level and at the position of the ISs to obtain a more realistic comparison between our theory and the recent Mach-Zehnder interferometry experiments. ${ }^{14,15}$

\section{COMPARISON OF THE $E$ FIELD AT DIFFERENT EDGE STATE MODELS}

In principle, the electron velocity at the edge of the 2DES or the electric field at the depleted region is not directly measured in the SPV experiments; instead, the slope of the potential profile is investigated as a function of the magnetic field deduced by the energy dependence of the SPV signal. On the other hand, in our calculations, we explicitly obtain the self-consistent potential, and by taking the derivative of the energy dispersion, we can directly calculate the electron velocity. In the previous section, by making use of the Thomas-Fermi approximation, we obtained the screened potential and claimed that the center coordinate dependent dispersion is given by Eq. (10). In the next step, we calculate the full screened potential $\left[V_{\mathrm{scr}}^{\mathrm{T} \neq 0, B \neq 0}(X)\right]$ and investigate its slope as a function of $B, T, c$ and $d$ as well as the long-range part of the disorder potential.

As a standard technique, $, 22,25,26$ we simulate the potential fluctuations generated by the disorder by imposing a modulation potential ${ }^{26}$ of the type

$$
V_{m}(x)=V_{0} \cos \left(k_{\lambda} x\right) \quad \text { with } k_{\lambda}=(\lambda+1 / 2) \pi / d
$$

as an additive contribution to the confinement potential. Here, $V_{0}$ is the modulation amplitude and $\lambda$ is an integer to preserve the boundary conditions.

In Fig. 5, we show the numerical derivative of $V_{\text {scr }}(x)$ at the chemical potential. Note that at $T \neq 0$, the Fermi energy is no longer equal to the chemical potential, and it has to be calculated for the given set of physical parameters. Here, we consider $\lambda=5$. The amplitude of the modulation is set such that, after screening, the potential variation is at the order of $5 \%-25 \%$ of $E_{F}^{0}$. As a rough estimate, we find that $V_{0}$ reduces 

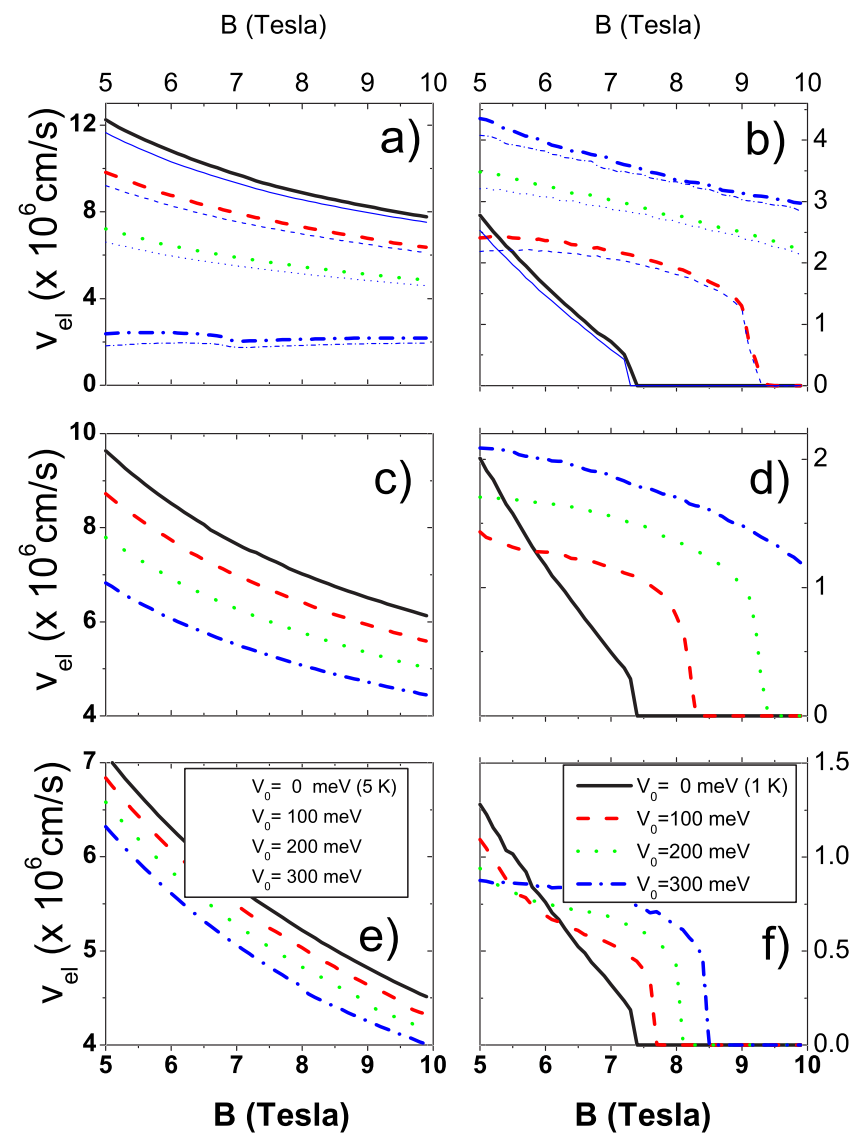

FIG. 5. (Color online) The slopes of the screened potential calculated at the Fermi level (left panel) and within the IS (right panel), considering characteristic (half) sample widths of $d=1 \mu \mathrm{m}$ (upper panel), $d=2 \mu \mathrm{m}$ (middle panel) and $d=5 \mu \mathrm{m}$ (lower panel). The electron temperatures are chosen to be $T=1 \mathrm{~K}$ (thick lines) and $T=5 \mathrm{~K}$ (thin lines) only in the upper panel.

by 3 orders of magnitude due to the dielectric screening [for GaAs, $\kappa=12.4]$, and electronic screening $[\epsilon(q) \sim 41$, see, e.g., the expression given in the caption of Fig. 2 of Ref. 9 and the related text). Regardless of the variation in $d, T$, and $V_{0}$, the slope of the screened potential obtained at the chemical potential exhibits the previously observed $F \propto B^{-1 / 2}$ form; except the case where the modulation is so strong that slope remains unaffected at $d=1 \mu \mathrm{m}$ with $V_{0}=300 \mathrm{meV}$. The hint to understand this exception is found in Fig. 5(b), where we show the slope calculated inside the IS at the position corresponding to $\nu(x)=2$. We see that the derivative of the potential also behaves similarly to the one obtained at the chemical potential, namely the inverse square root form, which indicates that the ISs are considerably narrow. Also, from the density profile calculated (not shown here; however, the results of a similar calculation can be found in Ref. 26), we see that, due to the strong modulation, the outermost IS is narrow and its effect is negligible; thus, the slope remains almost insensitive to the change in the $B$ field on this scale. An interesting comparison concerning the sample widths reveals that the narrower the sample is, the stronger the slope. Hence, in the Mach-Zehnder experiments and also considering the fact that the measurements are performed at an inter- mediate magnetic field strength $(B \sim 2.5-4.5 \mathrm{~T})$ and narrow samples $(d \sim 1 \mu \mathrm{m})$, the assumption of a constant velocity independent of $B$ is not realistic. We observe that the disorder potential does not affect this general behavior as long as the dominating scattering processes come from the edges of the sample. Introducing disorder obviously results in density fluctuations, which can be screened by the 2DES if the system is compressible (far from integer filling factors), and the conclusion is the opposite if the Landau levels are fully occupied. We consider a situation such that the magnetic field is tuned to an interval where the average filling factor becomes close to an (even) integer. In this situation, a large IS is formed at the bulk (without modulation) and split into several ribbons (as observed in Fig. 2 of Ref. 22), and the effect of these incompressible ribbons on the slope at the edge is marginal. This is seen in the left panel of Fig. 5, where we examine the behavior of the derivative comparing $V_{0}=0$ and $V_{0} \neq 0$. In the unmodulated case, the slope drops linearly with increasing $B$, until a large IS is formed at the bulk (e.g., in Fig. 5(f), $B \sim 7.3$ T). The wide strip disappears when the magnetic field strength is strong enough so that the Fermi level is pinned to the lowest Landau level, $B$ $\sim 7.45 \mathrm{~T}$. For the modulated case, the derivative decreases also linearly, with a smaller slope. However, this linear region is larger compared to the unmodulated case, e.g., in Fig. $5(\mathrm{~d})$, up to $B \sim 7.8 \mathrm{~T}$ for $V_{0}=100 \mathrm{meV}$ and $B \sim 9.0 \mathrm{~T}$ for $V_{0}=200 \mathrm{meV}$. Depending strongly on the modulation amplitude, the rapid decay of the slope due to the formation of a large bulk IS is observed in a relatively narrow $B$ interval. The "linear slope regime" is observed for all considered sample widths; however, for narrow samples, the $B$ interval is larger for higher modulation amplitudes. This indicates that for high mobility samples, where the long-range part of the disorder potential is well screened, ${ }^{22}$ the linear regime will be observed in a narrow $B$ interval. From the above discussion we conclude that the electron velocity on the ISs presumes a linear $B$ field dependence. At this point, we find it useful to make a connection between our results and the Mach-Zehnder-type samples. These samples have intermediate mobility and are relatively narrow. We have shown that the electron velocity calculated at the chemical potential decreases like an inverse square root of the $B$ field, and the assumption of constant velocity is not applicable. If the current is carried by the ISs, assuming a constant $v_{\mathrm{el}}$ in the magnetic field interval where the interference pattern is observed is still irrelevant. Recently, it has been shown theoretically that, ${ }^{27}$ within the screening picture of integer quantized Hall effect, the interference can be observed only in a narrow magnetic field interval within the plateau regime at high mobilities. The boundaries to observe interference pattern is estimated such that two separated ISs should be formed [similar to $B<8 T$ of Fig. 6(f)], which are larger than the Fermi wave length $(B>6 T)$. This interval coincides with the linear velocity regime shown above. Therefore, we support the idea ${ }^{15}$ that the phase of the electron calculated within the single particle picture should also be reconsidered form the interaction point of view as presented in this work. So far, we have examined the magnetic field dependence of the slope of the screened potential at the Fermi level and within the ISs. We have found that, depending on the $B$ 

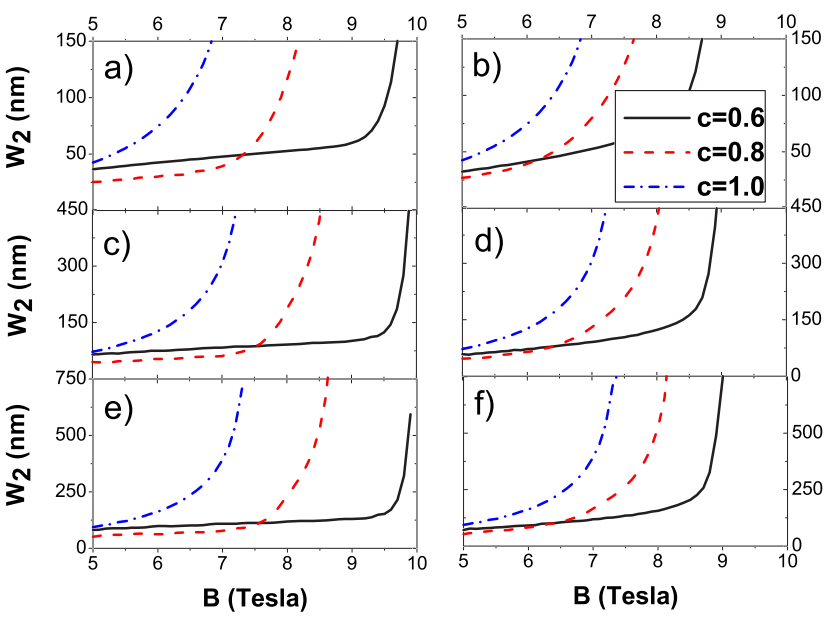

FIG. 6. (Color online) The sample width dependence of the IS thickness for $\nu(x)=2$. Calculations are performed at $1 \mathrm{~K}$ for three characteristic steepness values considering etched (left panel) and functionally doped (right panel) samples. Widths of the samples are selected to be $d=1 \mu \mathrm{m}$ (top) $d=3 \mu \mathrm{m}$ (middle), and $d=5 \mu \mathrm{m}$ (bottom), whereas the electron depleted strips are fixed to be $10 \%$ of $d$.

strength, the electron velocity exhibits different behaviors depending on where the slope is calculated. If it is assumed that the current flows from the Landauer-Büttiker-type edge states, the velocity takes the form $B^{-1 / 2}$. If the current is carried by the ISs, the velocity drops linearly in the case of two separate ISs and is highly nonlinear in the presence of a large IS in the bulk. Next, we discuss the extent of the ISs depending on the magnetic field and steepness of the confinement potential considering different sample widths.

\section{FORMATION OF INCOMPRESSIBLE STRIP}

The long-standing question of "where the current flows" in the quantum Hall bar systems has been addressed in many different theoretical works. ${ }^{10,11,28-31}$ In an early paper by Chang, ${ }^{30}$ it was stated that the current is confined to the ISs where the potential drops. This conjecture was supported by Fogler and Shklovskii, ${ }^{32}$ in which they calculated (at zero temperature) the magnetoresistance coefficients within the "electrostatic approximation" and the self-consistent treatment of the potential and density distribution was left unresolved. In a recent model ${ }^{10,11}$ using a local version of the Ohm's law, it was shown explicitly that the external current is confined in the ISs where the longitudinal resistivity vanishes, i.e., $\rho_{\ell}(x)=0$. This novel approach brought a quantitative explanation to many interesting aspects of the integer quantized Hall effect, among which are the high reproducibility of the very accurate quantized Hall plateaus, the transition between the zero states and the description of the local current distribution. This model is based on the formation (and disappearance) of the ISs, and now we concentrate on their widths taking into account different edge profiles and sample widths.

In Fig. 6, we plot the widths of the ISs $\left(W_{2}\right.$, for local filling factor of 2) against the magnetic field strength considering different sample properties. For the constant donor dis- tribution $(c=1)$, we see that the sample width has no influence on the $B$ dependence of the width of the strips, whereas $W_{2}$ increases by increasing the sample width, as expected. Note that, since the variation of the self-consistent potential at the IS is $\hbar \omega_{c}$, the slope is calculated simply by dividing this variation by $W_{2}$. As a direct consequence, the slope becomes small when $W_{2}$ becomes large. At the first glance, for intermediate steepness $(c=0.8)$, the functional form of the inhomogeneous donor distribution, i.e., $\rho_{1}(x)$ or $\rho_{2}(x)$, has no influence on $W_{2}$. However, the linear velocity regime is much more extended for the etched samples than that of the doped ones for relatively large sample widths $(d \gtrsim 3 \mu \mathrm{m})$. This feature is more pronounced for $c=0.6$, i.e., for the steeper edge profile, and the linear (velocity) regime is observed in a larger magnetic field interval compared to other steepness parameters. The observed difference is due to the extent of the electron poor region, given that the etched samples provide a steeper edge potential profile [see the discussion of Fig. 4(a)]. The slope of the linear regime is smaller for the etched sample and a smoother transition to the nonlinear regime is observed for the doped edge profile, whereas the functional form of the donor distribution seems to show no important difference for different sample widths.

As a final remark on the IS widths, we would like to recall the findings of Güven and Gerhardts ${ }^{10}$ where the high current regime was also investigated. It was shown that a large imposed current leads to a broadening of the ISs on one side of the sample, hence a change in the slope, which was also supported by the experiments. ${ }^{5}$ This result shows that there is a relation between the amplitude of the imposed current and the average electron velocity inside the ISs. We believe that the investigation of the out-of-the-linear response regime will improve our understanding of the Mach-Zehnder type of interferometer experiment. Our preliminary results show that the widths of the ISs increase linearly by increasing the amplitude of the applied current.

\section{SUMMARY}

In summary, we have calculated the slope of the selfconsistent potential, within the Thomas-Fermi-Poisson theory of screening. We considered two different pictures of edge states, namely, the single particle and the incompressible states, to obtain electron velocities in the presence of a strong perpendicular magnetic field. We have systematically investigated the effect of the sample properties such as the sample width, edge profile, and disorder potential on the electron velocities.

We first obtained a functional form of the $v_{\mathrm{el}}$ and the electric field depending on the magnetic field strength, without taking into account the formation of the ISs, and considering only the Landau quantization. It is shown that the interpretation of the SPV experiments ${ }^{13}$ strongly contradicts with our results and also with other experiments. ${ }^{19} \mathrm{We}$ found that the slope of the self-consistent potential changes as $\sim B^{-1 / 2}$, whereas the electric field at the edge behaves as $F$ $\propto B^{1 / 2}$. We also concluded that assuming a constant $v_{\mathrm{el}}$ may lead to discrepancies in analyzing the results of Mach-Zehnder-interferometer-type ${ }^{14,15}$ experiments. 
Secondly, by evaluating the full self-consistent potential, we were able to obtain the electron velocities at the Fermi level and within the ISs. We found that the full selfconsistent results coincide with our semiconsistent findings pointing to the inverse square root dependence of $v_{\mathrm{el}}$ within the single particle picture. The slope of the fully screened potential calculated at the ISs, however, exhibits two different regimes of magnetic field. These two regimes are identified by the dependence of the electron velocity on the magnetic field, which is linear in one regime and nonlinear in the other.

Our results indicate that, in narrow Hall bar geometries with intermediate mobilities, the edge profile becomes very important in determining the electron velocity for both the Landauer-Büttiker or the IS-type edge states. It appears to us that re-examining the results of Mach-Zehnder interferometer ${ }^{14,15}$ experiments from self-consistent point of view will thus be helpful in understanding the underlying physics of the obtained interference patterns.

\section{ACKNOWLEDGMENTS}

We would like to thank R. R. Gerhardts for his support and fruitful lectures on screening theory, which, in fact, enabled us to understand the basics. This work was financially supported by SFB 631, TÜBITAK Grant No. 105T110, and Trakya University research fund under project No. TÜBAP739-754-759. The authors are also grateful to the Institute of Theoretical and Applied Physics, Marmaris, Turkey for partial support where part of this work was carried out.
${ }^{1}$ R. J. F. van Haren, F. A. P. Blom, and J. H. Wolter, Phys. Rev. Lett. 74, 1198 (1995).

${ }^{2}$ A. Lorke, J. P. Kotthaus, J. H. English, and A. C. Gossard, Phys. Rev. B 53, 1054 (1996).

${ }^{3}$ W. Dietsche, K. von Klitzing, and K. Ploog, Surf. Sci. 361/362, 289 (1996).

${ }^{4}$ E. Ahlswede, P. Weitz, J. Weis, K. von Klitzing, and K. Eberl, Physica B 298, 562 (2001).

${ }^{5}$ E. Ahlswede, J. Weis, K. von Klitzing, and K. Eberl, Physica E (Amsterdam) 12, 165 (2002).

${ }^{6}$ S. Ilani, A. Yacoby, D. Mahalu, and H. Shtrikman, Phys. Rev. Lett. 84, 3133 (2000).

${ }^{7}$ S. Ilani, J. Martin, E. Teitelbaum, J. H. Smet, D. Mahalu, V. Umansky, and A. Yacoby, Nature (London) 427, 328 (2004).

${ }^{8}$ D. B. Chklovskii, B. I. Shklovskii, and L. I. Glazman, Phys. Rev. B 46, 4026 (1992).

${ }^{9}$ A. Siddiki and R. R. Gerhardts, Phys. Rev. B 68, 125315 (2003).

${ }^{10}$ K. Güven and R. R. Gerhardts, Phys. Rev. B 67, 115327 (2003).

${ }^{11}$ A. Siddiki and R. R. Gerhardts, Phys. Rev. B 70, 195335 (2004).

${ }^{12}$ A. Siddiki and R. R. Gerhardts, Int. J. Mod. Phys. B 18, 3541 (2004)

${ }^{13}$ B. Karmakar and B. M. Arora, Pramana, J. Phys. 67, 191 (2006).

${ }^{14}$ Y. Ji, Y. Chung, D. Sprinzak, M. Heiblum, D. Mahalu, and H. Shtrikman, Nature (London) 422, 415 (2003).

${ }^{15}$ I. Neder, M. Heiblum, Y. Levinson, D. Mahalu, and V. Umansky, Phys. Rev. Lett. 96, 016804 (2006).

${ }^{16}$ D. B. Chklovskii, K. A. Matveev, and B. I. Shklovskii, Phys. Rev.
B 47, 12605 (1993).

${ }^{17}$ U. Wulf, V. Gudmundsson, and R. R. Gerhardts, Phys. Rev. B 38, 4218 (1988).

${ }^{18}$ J. H. Oh and R. R. Gerhardts, Phys. Rev. B 56, 13519 (1997).

${ }^{19}$ M. Huber, M. Grayson, M. Rother, W. Biberacher, W. Wegscheider, and G. Abstreiter, Phys. Rev. Lett. 94, 016805 (2005).

${ }^{20}$ D. B. Chklovskii and P. A. Lee, Phys. Rev. B 48, 18060 (1993).

${ }^{21}$ Y. Y. Wei, J. Weis, K. v. Klitzing, and K. Eberl, Phys. Rev. Lett. 81, 1674 (1998).

${ }^{22}$ A. Siddiki and R. R. Gerhardts, Int. J. Mod. Phys. B 21, 1362 (2007).

${ }^{23}$ M. Büttiker, Phys. Rev. B 38, 9375 (1988).

${ }^{24}$ J. H. Davies, The Physics of Low-Dimensional Semiconductors (Cambridge University Press, New York, 1998).

${ }^{25}$ A. Siddiki, S. Kraus, and R. R. Gerhardts, Physica E (Amsterdam) 34, 136 (2006).

${ }^{26}$ A. Siddiki and R. R. Gerhardts, in Proceeding of the 15th International Conference on High Magnetic Fields in Semiconductor Physics, edited by A. R. Long and J. H. Davies (IOP, Bristol, 2002).

${ }^{27}$ A. Siddiki and F. Marquardt, Phys. Rev. B 75, 045325 (2007).

${ }^{28}$ M. Büttiker, Phys. Rev. Lett. 57, 1761 (1986).

${ }^{29}$ N. R. Cooper and J. T. Chalker, Phys. Rev. B 48, 4530 (1993).

${ }^{30}$ A. M. Chang, Solid State Commun. 74, 871 (1990).

${ }^{31}$ J. H. Han and D. J. Thouless, Phys. Rev. B 55, R1926 (1997).

${ }^{32}$ M. M. Fogler and B. I. Shklovskii, Phys. Rev. B 50, 1656 (1994). 